\title{
Multisystem Inflammatory Syndrome in Children Associated with COVID-19: A Review with an Emphasis on Mucocutaneous and Kawasaki Disease-Like Findings
}

\author{
Angelo Valerio Marzano ${ }^{a, b}$ Nicoletta Cassano ${ }^{c}$ Chiara Moltrasio $^{a, d}$ \\ Lucio Verdoni ${ }^{e}$ Giovanni Genovese ${ }^{a, b}$ Gino Antonio Vena ${ }^{c}$ \\ aDermatology Unit, Fondazione IRCCS Ca' Granda Ospedale Maggiore Policlinico, Milan, Italy; bepartment of \\ Pathophysiology and Transplantation, Università degli Studi di Milano, Milan, Italy; ${ }^{C}$ Dermatology and Venereology \\ Private Practice, Bari/Barletta, Italy; ${ }^{\mathrm{d}}$ Department of Medical Surgical and Health Sciences, University of Trieste, \\ Trieste, Italy; ePaediatric Department, Hospital Papa Giovanni XXIII, Bergamo, Italy
}

\section{Keywords}

COVID-19.SARS-CoV-2 $\cdot$ Multisystem inflammatory

syndrome in children · Kawasaki disease

\begin{abstract}
Background: COronaVIrus Disease 2019 (COVID-19) affects children with less severe symptoms than adults. However, severe COVID-19 paediatric cases are increasingly reported, including patients with Kawasaki disease (KD) or a multisystem inflammatory syndrome (MIS-C) that can present with features resembling KD. Summary: MIS-C is an emerging severe paediatric syndrome associated with COVID-19 that can show overlapping features of KD, KD shock syndrome, and toxic shock syndrome. MIS-C might be an inflammatory disease distinct from KD resulting from an exaggerated immune response. A high prevalence of mucocutaneous manifestations - in addition to gastrointestinal and cardiovascular involvements - was found in MIS-C. The most frequent mucocutaneous findings were conjunctivitis and rash, often described as macular and/or papular or polymorphous. In this article, we present a brief overview of MIS-C with an emphasis on mucocutaneous findings and the relationship with $\mathrm{KD}$.

(c) 2021 S. Karger AG, Basel
\end{abstract}

\section{Introduction}

COronaVIrus Disease 2019 (COVID-19) caused by severe acute respiratory syndrome coronavirus 2 (SARS$\mathrm{CoV}-2$ ) has led to a global pandemic, affecting individuals of all ages. However, paediatric cases are usually much less severe than adult cases [1]. The reasons for this difference are unknown, although hypothetical explanations include partial protective role of antibodies against other human coronaviruses responsible for infections during early childhood, lower prevalence of relevant comorbidities, different immunological responses, as well as differential expression of the angiotensin converting enzyme- 2 receptor, that is the primary target receptor for SARS-CoV-2 [1].

Nonetheless, with the rapid escalation of COVID-19 across the globe, severe paediatric cases, also requiring critical care, are increasingly reported, although an overall benign course is still observed in most children and adolescents and mortality rates remain much lower than those found in adults.

A.V.M. and N.C. contributed equally to this work. G.G. and G.A.V. contributed equally to this work karger@karger.com

www.karger.com/drm

Karger'

$$
\text { 兴 }
$$

(c) 2021 S. Karger AG, Base
Correspondence to:

Angelo Valerio Marzano, angelo.marzano@ unimi.it 


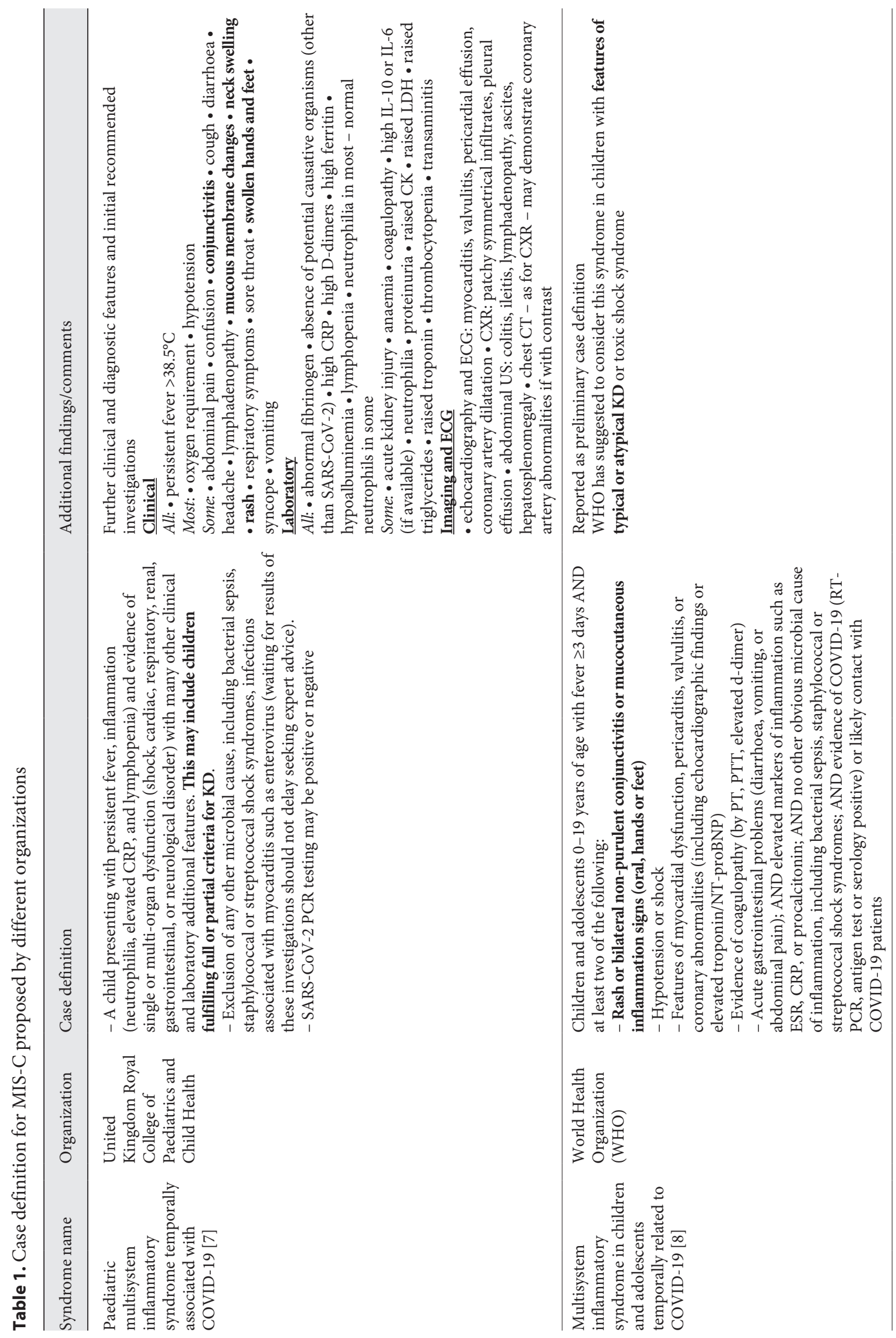




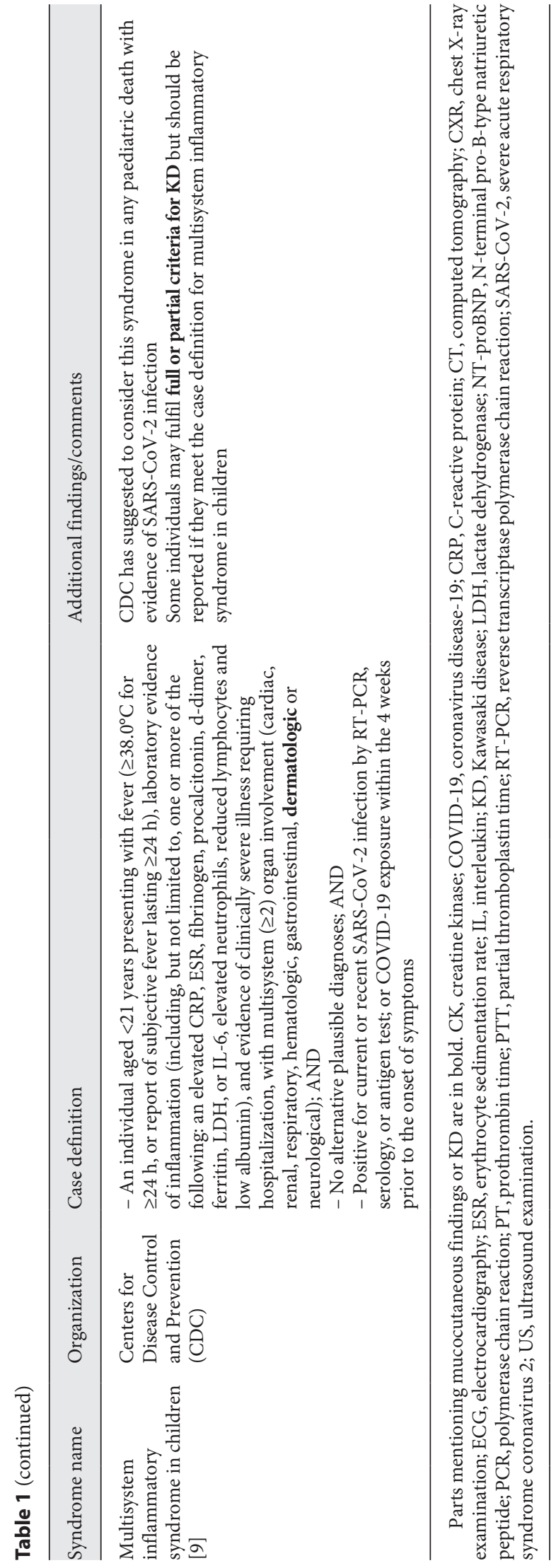

Multisystem Inflammatory Syndrome in Children
Since April 2020, paediatric cases of Kawasaki disease (KD) or Kawasaki-like disease possibly related to SARSCoV-2 infection have been observed [2-4]. The association of SARS-CoV-2 infection with KD has not been regarded as unexpected since viruses have been supposed to be possible triggers of $\mathrm{KD}$ [2]. Peculiar clinical presentations however emerged from the first reports of $\mathrm{CO}$ VID-19-related Kawasaki-like disease, depicting a hyperinflammatory syndrome with multiorgan dysfunction, frequent myocardial involvement, possible signs of macrophage activation syndrome (MAS), features reminiscent of atypical KD, KD shock syndrome or toxic shock syndrome, and prominent gastrointestinal but not respiratory symptoms [3-5].

Since then, authors from several other countries have also reported patients exhibiting similar features and described an unexpected outbreak of cases presenting with a serious multisystem inflammatory condition, sometimes displaying KD features [5, 6]. Various definitions of this clinical entity have been coined, including "Kawasaki-like syndrome/disease," "paediatric multisystem inflammatory syndrome temporally associated with COVID-19," and "multisystem inflammatory syndrome in children" (MIS-C). Case definition for this condition, which will be abbreviated as MIS-C throughout the text, has been proposed by different organizations (Table 1) [7-9].

The peak of the epidemic curve of MIS-C cases followed the peak of COVID-19 with an interval of approximately 4-6 weeks, suggesting that MIS-C could be a delayed post-infectious manifestation $[5,10]$. A recent report further supports this link, showing a strong lagged relationship between the rise and fall of MIS-C cases and the rise and fall of the first wave of the COVID-19 epidemic in France [11]. Interestingly, there were no reports of syndromes similar to MIS-C during the first phase of COVID-19 pandemic in Asia, even in countries where $\mathrm{KD}$ has the highest prevalence rates, suggesting the involvement of genetic factors in this discrepancy $[11,12]$.

Pathophysiology of MIS-C is still unclear and further research is needed for a better understanding of pathomechanisms and therapeutic implications. MIS-C is thought to be characterized by a cytokine storm involving innate and adaptive immune cells [10], and SARSCoV-2 might act either as a trigger or as an immunemodulating factor [11]. Possible mechanisms include antibody or T-cell recognition of self-antigens resulting in autoantibodies or autoreactive T cells, antibody or T-cell recognition of viral antigens expressed on infected cells, formation of immune complexes, and activation of a hy- 
Table 2. Diagnostic features of KD with description of mucocutaneous findings

Diagnosis of complete KD (also termed typical or classic KD) is based on the presence of $\geq 5$ days of fever AND the presence of $\geq 4$ of the 5 principal clinical features:

1. Changes of the lips and oral cavity They appear in the acute phase and comprise labial erythema, dryness, fissuring, peeling, cracking, and bleeding, "strawberry tongue" with erythema and prominent papillae and erythema of the oral and pharyngeal mucosa

2. Bilateral bulbar non-exudative It usually begins shortly after fever onset and often spares the limbus conjunctival injection

3. Cutaneous rash It generally appears during the first few days of the acute febrile phase

Described as polymorphous, varying from macular, maculopapular, or morbilliform to erythroderma or erythema multiforme-like. Less common presentations are urticarial, micropustular, or psoriasiform eruptions, whereas vesicular, bullous, or petechial lesions are not consistent with KD diagnosis. Most commonly, there is a diffuse maculopapular eruption that starts on the trunk and spreads over the next few days to involve the extremities. An accentuation of erythema over the perineal area with early desquamation seems to be a typical sign

4. Distinctive manifestations on the Erythema and oedema/painful induration of the hands and feet in acute phase extremities Periungual desquamation of the fingers and toes usually appears in the subacute phase, within $2-3$ weeks after fever onset, and may extend to the palms and soles

5. Cervical lymphadenopathy Usually unilateral $(\geq 1.5 \mathrm{~cm}$ diameter)

In the presence of $\geq 4$ principal clinical criteria, particularly if erythema and oedema of the hands and feet are present, the diagnosis may be made with only 4 days of fever.

Diagnosis of incomplete (atypical) KD should be considered in:

- infants or children with $\geq 7$ days of fever not otherwise explained

- in infants or children with prolonged unexplained fever, fewer than 4 of the principal clinical criteria, and compatible laboratory or echocardiographic findings

Coronary artery abnormalities are important to support the diagnosis in case of atypical KD. However, a normal echocardiogram in the first week of illness does not rule out the diagnosis of $\mathrm{KD}$, as coronary artery dilatation is usually not detected by echocardiography until after the first week of illness.

Laboratory tests, even if not specific, may give diagnostic support especially for patients with non-classic but suggestive clinical features. Typical laboratory findings may include normal or elevated WBC count with neutrophil preponderance and high levels of acute phase reactants, such as CRP and ESR, during the acute phase. Anaemia, hyponatremia, hypoalbuminemia, elevated serum liver enzymes, and sterile pyuria can be present. In the second week after fever onset, thrombocytosis is frequently observed.

The systemic inflammation involving the medium-sized arteries of multiple organs and tissues during the acute febrile phase is responsible for the development of many additional clinical findings, such as hepatitis, interstitial pneumonitis, abdominal pain, vomiting, diarrhoea, gallbladder hydrops, pancreatitis, aseptic meningitis, irritability, myocarditis, pericarditis, valvulitis, pyuria, and lymphadenopathy. 16].

CRP, C-reactive protein; ESR, erythrocyte sedimentation rate; KD, Kawasaki disease; WBC, white blood cell. Information obtained from [13, 15, and

perinflammatory response induced by viral superantigen fragments $[5,12]$. The role of other factors, such as genetic predisposition, dysfunctional microbiota, and vitamin D deficiency, has also been hypothesized $[6,12]$.

\section{KD and Main Differences between MIS-C and KD}

$\mathrm{KD}$ is an acute systemic vasculitic syndrome primarily affecting children below the age of 5 that involves small and medium-sized vessels with predilection for the coronary arteries. KD is usually a self-limiting disease, but prompt management is fundamental for the prevention of cardiac sequelae [13]. Pathogenic mechanisms have been linked to an exaggerated immunological response to environmental or infectious triggers in genetically sus- ceptible children, and a viral aetiology has been suggested [14].

In the absence of specific tests, the diagnosis of $\mathrm{KD}$ relies on its clinical manifestations (Table 2) $[13,15,16]$.

A severe form of $\mathrm{KD}$, named $\mathrm{KD}$ shock syndrome, is characterized by hypotension/shock during the acute phase and is associated with a greater risk of cardiac dysfunction, coronary artery abnormalities, and resistance to intravenous immunoglobulins (IVIg), as well as a more pronounced increase in inflammatory markers [13]. MAS is a rare life-threatening complication of $\mathrm{KD}$, consisting in an acute and rapidly progressive hyperinflammatory reaction that is often associated with refractoriness to IVIg [13]. 
The clinical spectrum of MIS-C frequently encompasses findings resembling $\mathrm{KD}$, particularly atypical forms.

In spite of some clinical overlap, MIS-C exhibits significant differences as confirmed by the comparison with historical cohorts of KD patients visited in the pre-COVID-19 period. For instance, patients with MIS-C or Kawasaki-like disease related to COVID-19 have been found to have an older age, a greater elevation of inflammatory markers, a higher frequency of gastrointestinal symptoms, myocardial dysfunction and shock, as well as a trend towards resistance to a single IVIg infusion and a greater risk for African or Hispanic origin [4-6, 10].

Therefore, specific epidemiological, clinical, and laboratory evidence appears to support the concept of a new systemic inflammatory disease resulting from an exaggerated immune response triggered by SARS-CoV-2 distinct from KD rather than a form of KD caused by SARS$\mathrm{CoV}-2$.

\section{General Clinical Features of MIS-C}

A great heterogeneity of studies recruiting MIS-C patients was found in the literature, particularly in terms of inclusion criteria and medical settings, as well as laboratory assessments and treatments administered [17]. The rates of cardiovascular, neurologic, and respiratory system involvement and severe symptoms requiring admission to intensive care units (ICUs) were variable as a consequence of differences in the inclusion criteria and settings between studies but ICU admissions appeared to involve a relevant proportion of MIS-C patients (nearly $70 \%)$ and a fatal outcome was documented in a few cases (approximately 1.7\%) [18, 19].

MIS-C is characterized by a hyperinflammatory state and a multisystem organ involvement with a preponderance of cardiovascular manifestations, including hemodynamic instability, ventricular dysfunction, and coronary artery abnormalities [6]. Some patients may present with features suggesting complete or incomplete $\mathrm{KD}$, toxic shock syndrome, KD shock syndrome, or MAS [3-5].

MIS-C can develop without prior typical symptoms of COVID-19 [11, 18]. Respiratory manifestations, which are predominant in COVID-19 patients, were found in a minority of MIS-C patients $[17,18]$. Lung involvement was not described as a prominent feature in most reports [19]. In some cohorts, the development of severe respiratory symptoms requiring intubation and extracorporeal membrane oxygenation appeared to be secondary to cardiovascular involvement [5].

Multisystem Inflammatory Syndrome in Children
Among the main manifestations, fever was virtually present in all cases as an integral part of any case definition [7-9]. Gastrointestinal symptoms are increasingly recognized as common presenting symptoms, potentially confusing the diagnosis of MIS-C with other gastrointestinal infections or inflammatory disorders and, occasionally, even conditions requiring urgent abdominal surgery $[18,19]$.

A systematic review including a total of 440 MIS-C cases (male 59\%; median age: 7.3-10 years) revealed a high prevalence of gastrointestinal (87\%), cutaneous/mucocutaneous (73\%), and cardiovascular (71\%) symptoms [17].

Whittaker et al. [20] gave a provisional differentiation into three main patterns of disease among hospitalized children: one group of children had persistent fever and elevated levels of inflammatory markers, without features of $\mathrm{KD}$, shock, or organ failure; a second group fulfilled the diagnostic criteria for KD; and a third had shock and clinical, echocardiographic, and laboratory signs of myocardial injury.

The analysis of 570 cases of MIS-C reported to Centers for Disease Control and Prevention (CDC) as of July 29, 2020 has led to identify three main classes, each corresponding to approximately one third of patients [21]. The first class with the highest number of involved organ systems, particularly cardiovascular (100\%) and gastrointestinal (97.5\%) systems, included patients with higher prevalence of abdominal pain, shock, myocarditis, lymphopenia, and markedly elevated inflammatory and cardiac biomarkers. Almost all of these patients had positive SARS-CoV-2 serology. In class $2,76 \%$ of patients had respiratory system involvement, suggesting that they might have suffered primarily from acute COVID-19 alone or combined with MIS-C rather than from true MIS-C. The frequency of SARS-CoV-2 reverse transcriptase-polymerase chain reaction (RT-PCR) positivity without seropositivity in this group (84\%) was significantly higher than that detected in the other two classes and mortality rate was the highest. Patients belonging to class 3 had the highest prevalence of rash (63\%) and mucocutaneous lesions (45\%) and more frequently fulfilled the criteria for complete $\mathrm{KD}(7 \%)$. Like $\mathrm{KD}$, MIS-C is a syndrome with multiple clinical presentations that lacks pathognomonic clues or specific diagnostic tests. It cannot be ruled out that some cases diagnosed as MIS-C might have had COVID-19 with "cytokine storm" [21]. However, many MIS-C patients across the various studies did not show signs of acute COVID-19 [12, 19].

A prospective observational study in 651 patients aged less than 19 years admitted to 138 hospitals in the UK

Dermatology 2022;238:35-43

DOI: $10.1159 / 000515449$ 
with laboratory-confirmed SARS-CoV-2 infection recognized subjects who met the criteria for MIS-C in both the acute phase of infection (RT-PCR positive) and postacute or convalescent phase of infection (antibody positive) groups [22].The latter group was found to be more frequently associated with non-white ethnicity and presence of muco-enteric symptoms (abdominal pain and conjunctivitis), whereas the acute group had more commonly respiratory symptoms. Three clusters of clinical phenotypes were identified in the whole paediatric cohort, consisting in discrete respiratory symptoms, systemic mucocutaneous-enteric symptoms, and, less commonly, neurological symptoms. The systemic mucocutaneous-enteric cluster presented with clinical features of the WHO preliminary case definition for MIS$\mathrm{C}$ [8], including rash, conjunctivitis, diarrhoea, vomiting, and abdominal pain, associated with headache, myalgia, sore throat, fatigue, and lymphadenopathy [22]. The two main "respiratory" and "systemic mucocutaneous-enteric" clinical clusters were not completely dichotomous, since minor overlap was observed between a sub-cluster of "fever, cough, and shortness of breath" and a sub-cluster of "vomiting, abdominal pain, diarrhoea, fatigue, and rash". The same study disclosed an association between obesity and MIS-C, especially in RT-PCR-positive patients. Moreover, compared with COVID-19 young patients without MIS-C, MIS-C patients were five times more likely to be admitted to critical care and more likely to receive intravenous corticosteroids, non-invasive and invasive ventilation, and inotropic support [22].

\section{Laboratory Findings in MIS-C Patients}

Laboratory confirmation of SARS-CoV-2 infection was demonstrated in variable proportions of patients, with positive results for RT-PCR testing detected in 13-69\% and for serology from 75 to $100 \%$ [17]. Moreover, the high rate of identification of antibodies with RT-PCR results that can be negative or indicative of a low viral load among MIS-C patients strongly supports that this syndrome could result from an abnormal immunological reaction that often develops when the virus is no longer detectable [17].

Laboratory investigations in the various studies consistently demonstrated that most cases had markedly elevated inflammatory markers at admission. A considerable proportion of MIS-C patients showed increased levels of D-dimer, fibrinogen, and interleukin-6, as well as lymphocytopenia, neutrophilia, hypoalbuminemia, anaemia, and thrombocytopenia [7-9, 17-19]. In the prospective observational cohort study performed by Swann et al. [22], children with MIS-C were found to be more likely to have a low platelet count than children with COVID-19 who did not have MIS-C.

Other findings included elevated lactate dehydrogenase levels, increase in transaminases, and signs of acute kidney injury. Highly elevated levels of cardiac damage/ heart failure markers, including troponin, brain natriuretic peptide (BNP), and N-terminal pro-BNP, were observed in many patients [17-19].

\section{Therapeutic Aspects of MIS-C}

Identification of MIS-C is important because of the risk of severe organ dysfunction requiring intensive care support, as well as the need of timely therapeutic management in order to control the hyperinflammatory state and prevent organ damage. Management of MIS-C implies the importance of multidisciplinary care and current strategies have been partially derived from the experience in $\mathrm{KD}$, toxic shock syndrome, and COVID-19-related inflammatory response, including supportive care, IVIg, corticosteroids, biological immunomodulators, antiplatelet therapy, and prophylactic anticoagulation $[5,19]$. Systematic reviews have demonstrated that IVIg was the most common medication (administered in nearly three-quarters of cases, and even more in patients with KD presentations), followed by vasoactive agents and corticosteroids $[17,18]$.

Although short-term survival is high, the long-term outcomes of children with MIS-C are unknown.

\section{Cutaneous and Mucosal Findings and KD-Like}

Features in MIS-C Patients

Mucosal and cutaneous findings similar to those observed in $\mathrm{KD}$, especially rash and conjunctivitis, were among the most common presentations of MIS-C. Conjunctivitis and rash were described in 52 and 56\%, respectively, of the 662 MIS-C cases included in the systematic review performed by Ahmed et al. [18].

A cutaneous/mucocutaneous involvement was estimated to occur in nearly three-quarters of MIS-C patients according to Abrams et al. [17] and in more than $80 \%$ of patients according to Young et al. [23]. The CDC report of 570 MIS-C cases documented the presence of cutaneous or mucocutaneous involvement in $71 \%$ of cases, with rash and conjunctival injection in 55 and $48 \%$ of cases, respectively [21].

The systematic review carried out by Kaushik et al. [19] summarized the reported frequency of findings suggestive of KD presentations as follows: rash in $58 \%$ of patients, conjunctival injection in $40 \%$, red cracked lips in $23 \%$, oedema over hands and feet in $13 \%$, strawberry tongue in $4.5 \%$, and cervical lymphadenitis in $4 \%$ of pa- 

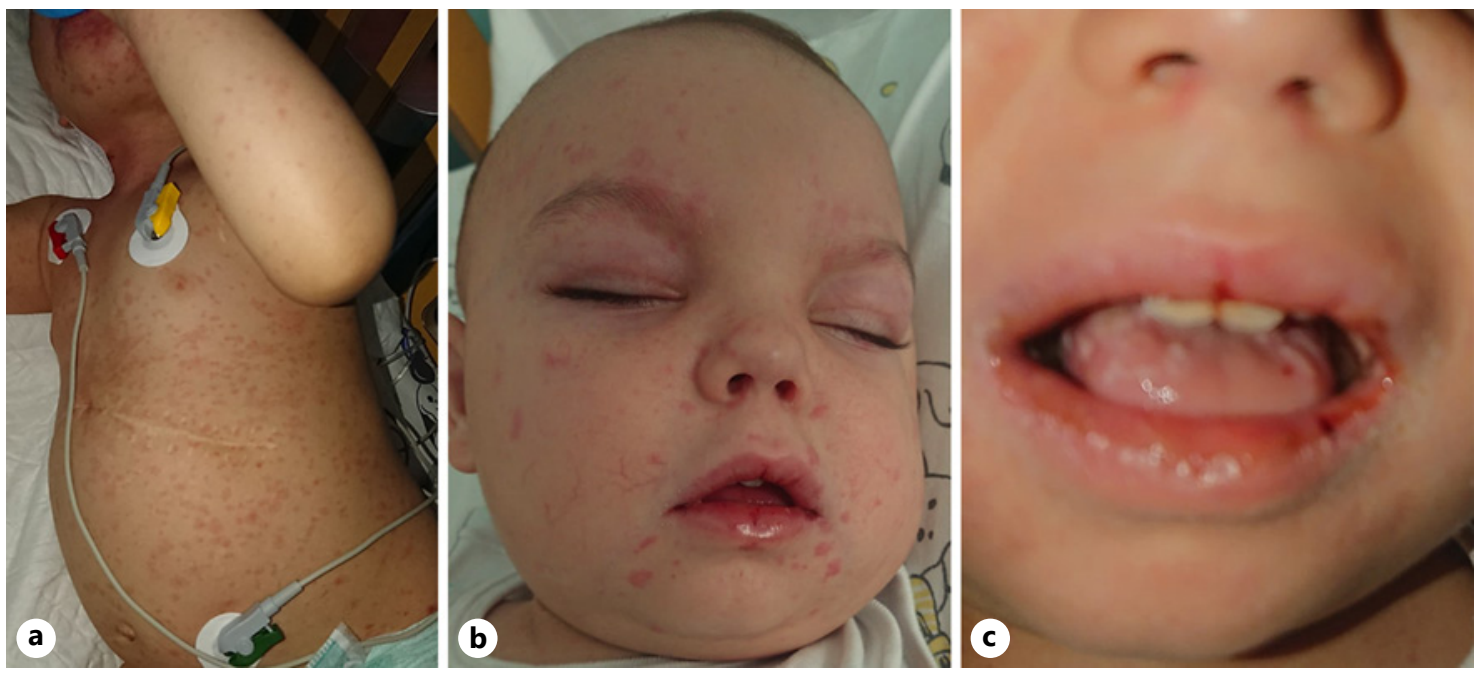

Fig. 1. Clinical features of MIS-C. Erythematous maculo-papular rash on the trunk (a) and face (b). Erosive lesions on the lips (c).

tients. Conjunctival injection in association with gastrointestinal manifestations was observed most frequently in children at presentation in studies from the USA [19].

The study carried out by Feldstein et al. [24] in 186 MIS-C patients demonstrated a variable frequency of mucocutaneous manifestations among age groups (79$80 \%$ in children aged $\leq 12$ years and $56 \%$ for older patients). Dufort et al. [25] also found in a cohort of 99 patients with MIS-C that the prevalence of cutaneous manifestations was highest among younger children, especially in those with age $0-5$ years.

No pathognomonic cutaneous or mucosal lesions have been associated with MIS-C so far. Rash and conjunctivitis are known to be common in viral syndromes and not specific for either KD or MIS-C.

Moreover, detailed descriptions of the mucocutaneous manifestations, including the onset or location, were not frequently reported in publications regarding patients with MIS-C [6]. However, various articles generally included mucosal and/or cutaneous lesions in the clinical spectrum observed during the baseline examination. Skin rash was frequently described as maculopapular (Fig. 1ac), rarely with a purpuric component $[6,20]$. When specified, conjunctivitis was defined as bilateral and non-exudative, as that observed in KD patients. Other mucocutaneous manifestations described with varying prevalence were erythema and/or oedema of the hands and feet, oral mucosa and lip changes (Fig. 1c), and facial or periorbital oedema $[6,21]$. Perineal or face desquamation have also been described [26]. Oral ulceration has been proposed as a possible early feature of MIS-C in some children followed by inflammatory changes in the intestinal tract [27].

Heterogenous morphologic patterns of mucocutaneous disease in MIS-C patients were recorded in a cohort study of prospectively and consecutively eligible patients aged less than 19 years who were admitted between May 11,2020 , and June 5, 2020 to a tertiary hospital located in New York [28]. In the MIS-C cohort, $47 \%$ of patients had rash and/or mucositis, including non-specific erythema, morbilliform eruption, retiform purpura, targetoid and urticarial lesions, along with lip fissuring or cracking (44\%), tongue papillitis (22\%), conjunctivitis (22\%), and acral oedema. Rash among MIS-C patients most frequently showed a peripheral distribution. The same study compared mucocutaneous findings in MIS-C patients and those in COVID-19 children and adolescents. Nonspecific erythema, morbilliform eruption, or lip mucositis did not differentiate MIS-C from COVID-19, whereas retiform purpura, targetoid or urticarial lesions, acral oedema or erythema, tongue papillitis, and conjunctivitis were seen only among MIS-C patients [28].

Interestingly, a potential prognostic value of rash in the setting of MIS-C was suggested. In fact, compared to MIS-C patients without rash, those with rash were shown to have lower levels of inflammatory markers, as well as less frequent paediatric ICU admission, shock, and requirement for invasive mechanical ventilation [28].

The frequency of diagnosing complete or incomplete $\mathrm{KD}$ among MIS- $\mathrm{C}$ patients appeared to be extremely variable among the studies, possibly because of differences in 
inclusion criteria and other study characteristics. For example, among the 570 cases reported to CDC [21], 5\% of patients met the criteria for complete $\mathrm{KD}$, whereas criteria for complete or incomplete $\mathrm{KD}$ were fulfilled by $36 \%$ of patients in the study of Dufort et al. [25] and 40\% of those examined by Feldstein et al. [24]. Whittaker et al. [20] found the fulfilment of the diagnostic criteria for $\mathrm{KD}$ in $12 \%$ of cases, and this proportion increased to $22 \%$ when coronary artery aneurysms were considered in patients with less than 4 criteria. In the study by Belhadjer et al. [29], who recruited patients with MIS-C and acute heart failure, clinical signs suggestive of $\mathrm{KD}$ were frequent, but none of the patients met criteria for classical KD. Among the 19 patients with MIS-C evaluated by Rekhtman et al. [28], none met the 2017 American Heart Association criteria for KD and very few met the criteria for incomplete $\mathrm{KD}$, even when coronary aneurysms were added to the diagnostic criteria.

Dufort et al. [25] demonstrated that diagnosis of complete or incomplete $\mathrm{KD}$ was less common in patients with $13-20$ years of age ( $12 \%$ vs. $48 \%$ in patients $0-5$ years of age and $43 \%$ in those $6-12$ years).

\section{Conclusions}

Progress is being made in understanding the puzzling clinical spectrum of COVID-19. A wide range of cutaneous manifestations have been described in patients with COVID-19 [30-32], whose pathomechanisms are still unknown. Some of these manifestations might be the direct consequence of the cytopathic effect of SARS-CoV-2 interacting with the skin, whereas others (paraviral eruptions) may result from the activation of the immune system triggered by the virus [33].

The recent recognition of MIS-C has raised great interest, and numerous efforts are currently being directed towards defining diagnostic criteria, prognostic factors, treatment approaches, and long-term effects.

The presence of features resembling $\mathrm{KD}$ is an intriguing aspect, and it cannot be ruled out that the research on this new entity might indirectly help better understanding KD itself.

Prompt recognition of MIS-C is important to control the hyperinflammatory state and prevent severe organ dysfunction and potentially fatal complications. Cutaneous and/or mucosal manifestations may occur as presenting symptoms in a relevant proportion of patients with MIS-C and may be important clues to suspect this syndrome in febrile children and adolescents during this pandemic period.

\section{Key Message}

Mucocutaneous findings are highly prevalent in multisystem inflammatory syndrome in children, an emerging severe paediatric syndrome associated with COVID-19.

\section{Conflict of Interest Statement}

The authors have no conflicts of interest to declare.

\section{Statement of Ethics}

Written informed consent for publication of clinical photographs of the patient was given by the parents of the patient.

\section{Funding Sources}

This paper did not receive any funding.

\section{Author Contributions}

Angelo Valerio Marzano and Nicoletta Cassano wrote the manuscript with the contribution of Giovanni Genovese and Chiara Moltrasio. Angelo Valerio Marzano, Gino Antonio Vena, and Lucio Verdoni supervised the work and revised the manuscript for critical revision for important intellectual content.

\section{References}

1 Rajapakse N, Dixit D. Human and novel coronavirus infections in children: a review. Paediatr Int Child Health. 2020:1-20.

2 Jones VG, Mills M, Suarez D, Hogan CA, Yeh D, Segal JD, et al. COVID-19 and Kawasaki Disease: Novel Virus and Novel Case. Hosp Pediatr. 2020;10(6):537-40.

3 Riphagen S, Gomez X, Gonzalez-Martinez C, Wilkinson N, Theocharis P. Hyperin- flammatory shock in children during COVID-19 pandemic. Lancet. 2020;395:16078.

4 Verdoni L, Mazza A, Gervasoni A, Martelli L, Ruggeri M, Ciuffreda M, et al. An outbreak of severe Kawasaki-like disease at the Italian epicentre of the SARS-CoV-2 epidemic: an observational cohort study. Lancet. 2020; 395(10239):1771-8.
5 Jiang L, Tang K, Levin M, Irfan O, Morris SK, Wilson PK, et al. COVID-19 and multisystem inflammatory syndrome in children and adolescents. Lancet Infect Dis. 2020;20(11):e276-88.

6 Lawrensia S, Henrina J, Wijaya E, Suciadi LP, Saboe A, et al. Pediatric Inflammatory Multisystem Syndrome Temporally Associated with SARS-CoV-2: a New Challenge amid the Pandemic. SN Compr Clin Med. 2020;2:1-9. 
7 Royal College of Paediatrics and Child Health. Guidance. Paediatric multisystem inflammatory syndrome temporally associated with COVID-19. Available from: https://www. rcpch.ac.uk/resources/guidance-paediatricmultisystem-inflammatory-syndrome-temporally-associated-covid-19-pims.

8 World Health Organization. Multisystem inflammatory syndrome in children and adolescents with COVID-19. Available from: https: //www.who.int/news-room/commentaries/ detail/multisystem-inflammatory-syndrome-in-children-and-adolescents-withcovid-19.

9 Centers for Disease Control and Prevention, Health Alert Network. Multisystem inflammatory syndrome in children (MIS-C) associated with coronavirus disease 2019 (COVID-19). Available from: https://emergency. cdc.gov/han/2020/han00432.asp.

10 Brodsky NN, Ramaswamy A, Lucas CL. The Mystery of MIS-C Post-SARS-CoV-2 Infection. Trends Microbiol. 2020;28(12):956-8.

11 Carbajal R, Lorrot M, Levy Y, Grimpel E, Lecarpentier T, Heritier S, et al. Multisystem inflammatory syndrome in children rose and fell with the first wave of the COVID-19 pandemic in France.

12 Buonsenso D, Riitano F, Valentini P. Pediatric Inflammatory Multisystem Syndrome Temporally Related With SARS-CoV-2: Immunological Similarities With Acute Rheumatic Fever and Toxic Shock Syndrome. Front Pediatr. 2020;8:574.

13 McCrindle BW, Rowley AH, Newburger JW, Burns JC, Bolger AF, Gewitz M, et al. Diagnosis, Treatment, and Long-Term Management of Kawasaki Disease: A Scientific Statement for Health Professionals From the American Heart Association. Circulation. 2019;140(5): e181-e184.[not available in pubmed,crossref]

14 Rowley AH, Shulman ST. The Epidemiology and Pathogenesis of Kawasaki Disease. Front Pediatr. 2018;6:374.

15 Marchesi A, Tarissi de Jacobis I, Rigante D, Rimini A, Malorni W, Corsello G, et al. Kawasaki disease: guidelines of the Italian Society of Pediatrics, part I - definition, epidemiology, etiopathogenesis, clinical expression and management of the acute phase. Ital J Pediatr. 2018;44(1):102.
16 Gupta A, Singh S. Kawasaki disease for dermatologists. Indian Dermatol Online J. 2016; 7(6):461-70.

17 Abrams JY, Godfred-Cato SE, Oster ME Chow EJ, Koumans EH, Bryant B, et al. Multisystem Inflammatory Syndrome in Children (MIS-C) Associated with SARS-CoV-2: A Systematic Review. J Pediatr. 2020;226:4554.

18 Ahmed M, Advani S, Moreira A, Zoretic S, Martinez J, Chorat K, et al. Multisystem inflammatory syndrome in children: A systematic review. EClinicalMedicine. 2020;26: 100527.

19 Kaushik A, Gupta S, Sood M, Mangla S, Seema S, Shinka V. A Systematic Review of Multisystem Inflammatory Syndrome in Children Associated With SARS-CoV-2 Infection. Pediatr Infect Dis J. 2020;39(11): e340-6.

20 Whittaker E, Bamford A, Kenny J, Kaforou $\mathrm{M}$, Jones CE, Shah P, et al. Clinical Characteristics of 58 Children With a Pediatric Inflammatory Multisystem Syndrome Temporally Associated With SARS-CoV-2. JAMA. 2020; 324(3):259-69.

21 Godfred-Cato S, Bryant B, Leung J, Oster ME, Conklin L, Abrams J, et al. COVID-19-Associated Multisystem Inflammatory Syndrome in Children - United States, March-July 2020. MMWR Morb Mortal Wkly Rep. 2020; 69(32):1074-80.

22 Swann OV, Holden KA, Turtle L, Egan C, Girvan M, Pritchard M, et al. Clinical characteristics of children and young people admitted to hospital with COVID-19 in United Kingdom: prospective multicentre observational cohort study. BMJ. 2020;370:m3249.

23 Young TK, Shaw KS, Shah JK, Noor A, Alperin RA, Ratner AJ, et al. Mucocutaneous Manifestations of Multisystem Inflammatory Syndrome in Children During the COVID-19 Pandemic. JAMA Dermatol. 2020 Dec 9: e204779.

24 Feldstein LR, Rose EB, Horwitz SM, Collins JP, Newhams M, Son MB, et al. Multisystem Inflammatory Syndrome in U.S. Children and Adolescents. N Engl J Med. 2020;383(4): $334-46$.
25 Dufort EM, Koumans EH, Chow EJ, Rosenthal E, Muse A, Rowlands J, et al. Multisystem Inflammatory Syndrome in Children in New York State. N Engl J Med. 2020;383(4):34758.

26 Toubiana J, Poirault C, Corsia A, Baiolle F, Fourgeaud J, Angoulvant F, et al. Kawasakilike multisystem inflammatory syndrome in children during the covid-19 pandemic in Paris, France: prospective observational study. BMJ. 2020;369:m2094.

27 Cant A, Bhujel N, Harrison M. Oral ulceration as presenting feature of paediatric inflammatory multisystem syndrome associated with COVID-19. Br J Oral Maxillofac Surg.

28 Rekhtman S, Tannenbaum R, Strunk A, Birabaharan M, Wright S, Garg A. Mucocutaneous Disease and Related Clinical Characteristics in Hospitalized Children and Adolescents with COVID-19 andmultisystem inflammatory syndrome in children. J Am Acad Dermatol. $2021 \mathrm{Feb} ; 84(2): 408-14$.

29 Belhadjer Z, Méot M, Bajolle F, Khraiche D, Legendre A, Abakka S, et al. Acute heart failure in multisystem inflammatory syndrome in children (MIS-C) in the context of global SARS-CoV-2 pandemic. Circulation. 2020; 142(5):429-36.

30 Galván Casas C, Català A, Carretero Hernández G, Rodríguez-Jiménez P, Fernández-Nieto $\mathrm{D}$, Rodríguez-Villa Lario A, et al. Classification of the cutaneous manifestations of COVID-19: a rapid prospective nationwide consensus study in Spain with 375 cases. Br J Dermatol. 2020;183(1):71-7.

31 Marzano AV, Cassano N, Genovese G, Moltrasio C, Vena GA. Cutaneous manifestations in patients with COVID-19: a preliminary review of an emerging issue. Br J Dermatol. 2020 Sep;183(3):431-42.

32 Genovese G, Moltrasio C, Berti E, Marzano AV. Skin Manifestations Associated with COVID-19: Current Knowledge and Future Perspectives. Dermatology. 2021;237(1):1-12.

33 Lipsker D. Paraviral eruptions in the era of COVID-19: Do some skin manifestations point to a natural resistance to SARS-CoV-2? Clin Dermatol. 2020 Nov - Dec;38(6):75761. 